\title{
SYNTHESIS AND CHARACTERIZATION OF SOME NEW TRANSITION METAL COMPLEXES WITH 2,5-di(4-AMINO PHENAZONYL) HEXANE
}

\author{
Abbas Ali Salih Al-Hamdani \\ Department of Chemistry, College of Science for Women, University of Baghdad.
}

\begin{abstract}
A new tetradentate $\mathrm{N}_{2} \mathrm{O}_{2}$ type Schiff base ligand 2,5-di(4-amino phenazonyl)hexane L was synthesized from the condensation of 4-aminophenazone and 2,5-hexanedone. Monomeric complexes of this ligand, of general formulae $[\mathrm{M}(\mathrm{L})] \mathrm{X}\left(\mathrm{M}-\mathrm{X}=\mathrm{Co}(\mathrm{II})-(\mathrm{OAc})_{2}, \mathrm{Ni}(\mathrm{II})-(\mathrm{OAc})_{2}\right.$, $\mathrm{Cu}(\mathrm{II})-(\mathrm{OAc})_{2}, \mathrm{VO}$ (II)-SO $\mathrm{SO}_{4}, \mathrm{Zn}(\mathrm{II})-\mathrm{SO}_{4}, \mathrm{Mn}$ (II)-Cl $\mathrm{Cl}_{2}$ or $\mathrm{Fe}(\mathrm{II})-\mathrm{Cl}_{2} ; \mathrm{OAc}=$ acetate $)$ are reported. The mode of bonding and overall geometry of the complexes were determined through I.R, UV-Vis, NMR spectral studies, magnetic moment measurements, elemental analysis, metal content and conductance measurement. These studies revealed tetrahedral geometries for the $\mathrm{Co}(\mathrm{II})$, $\mathrm{Ni}(\mathrm{II}), \mathrm{Mn}(\mathrm{II}), \mathrm{Fe}(\mathrm{II})$ and $\mathrm{Zn}(\mathrm{II})$ complexes, square pyramidal for $\mathrm{VO}(\mathrm{II})$ complex and square planar for $\mathrm{Cu}(\mathrm{II})$ complex. The study of complex formation via molar ratio in DMF solution was investigated and the results were compeer to those found in the solid complexes with a ratio of (M: L) as $(1: 1)$
\end{abstract}

Keywork: 4-aminophenazone, 2,5-hexanedone, Schiff base complexes.

\section{Introduction}

Schiff bases from 2,5-hexanedione have often been used as chelating ligands in the field of coordination chemistry. Recently, liquid crystals researchers have made a significant revelation that the introduction of a lateral polar carbonyl group will enhance the molecular polarizabilty as well as stabilize the liquid crystalline compounds ${ }^{(1)}$.

Diketone compounds take a role in creating various fragrances and compounds to form complexes with many transition metal ions. These compounds are readily soluble in inorganic solvents. Transition metal complexes with tetradentate Schiff base ligands have been extensively investigated as catalysts for a number of organic redox reaction and electrochemical reduction processes ${ }^{(2)}$. Cyclic voltammetry has been a useful tool to investigate the mechanisms of catalysis by Schiff base metal complexes as well as to study the structure reactivity relationships in these compounds ${ }^{(3-5)}$.

Phenazone derivatives are reported to exhibit analgesic and anti-inflammatory effects ${ }^{(6,7)}$, antiviral $^{(8)}$ antibacterial ${ }^{(9)}$ activities. Furthermore, they have been used as hair color additives ${ }^{(10)}$ and to potentiate the local anesthetic effect of lignocaine. ${ }^{(11)}$
In the present paper, $\mathrm{Co}(\mathrm{II}), \mathrm{Ni}(\mathrm{II}), \mathrm{VO}(\mathrm{II})$, $\mathrm{Mn}$ (II), $\mathrm{Fe}(\mathrm{II}), \mathrm{Zn}$ (II) and $\mathrm{Cu}(\mathrm{II})$ complexes with Schiff base derived from 4-amino phenazone and 2,5-hexanedione are reported. These compounds have been characterized in the basis of analysis of the Schiff bases liquid and solid complexes. ${ }^{1}$ HNMR, ${ }^{13} \mathrm{CNMR}$, elemental analysis (C.H.N and M) whereas: $\mathrm{M}=\mathrm{Co}, \mathrm{Ni}, \mathrm{V}, \mathrm{Mn}, \mathrm{Fe}, \mathrm{Zn}$ and $\mathrm{Cu}$, magnetic measurements, UV-Visible, FTIR Spectra, Conductivity Measurement and Molar Ratio.

\section{Experimental}

All the chemicals such as the metal salts $\left[\mathrm{Co}(\mathrm{OAc})_{2} . \quad 4 \mathrm{H}_{2} \mathrm{O}, \quad \mathrm{VOSO}_{4}, \mathrm{ZnSO}_{4}\right.$, $\mathrm{MnCl}_{2} .4 \mathrm{H}_{2} \mathrm{O}, \mathrm{FeCl}_{2}, \mathrm{Ni}(\mathrm{OAc})_{2} 6 \mathrm{H}_{2} \mathrm{Cu}(\mathrm{OAc})_{2}$. $\mathrm{H}_{2} \mathrm{O}$ ] and solvents dimetylformamide, Ethanol, Dimethylsulfoxide, diethyl ether and methanol were obtained from Merk and 2,5-hexandione and 4 -amino phenazone were obtained from Fluke and used without further purification. Conductivity measure- ments of $10^{-3} \mathrm{M}$ solutions of the complexes were recorded in DMF at $25 \mathrm{C}^{\circ}$ using Hand-Held Meter LF 330. The electronic spectra of the complexes in $\mathrm{UV}$-Vis region were recorded in DMF solution using Shimadzu Model 160 UV-Visible Spectrophotometer. IR-spectra CsI discs in the range (4000-250) $\mathrm{cm}^{-1}$ were obtained using a Shimadzu, FTIR-8400 S Fourier Transform Infrared Spectrophotometer. Atomic 
Absorption Data were obtained via using (Shimadzu A.A-160) Atomic absorption/ flame Emission Spectrophotometer. Magnetic properties were recorded via using (Balance Magnetic Susceptibility Model MSR-MKi). Furthermore, Melting Points were measured via using (Gallen Kamp Melting Point).

${ }^{1} \mathrm{HNMR},{ }^{13} \mathrm{CNMR}$ spectra were recorded via using Bruker $400 \mathrm{MHz}$ Spectrophotometer Elemental Analysis for $(\mathrm{C}, \mathrm{H}$ and $\mathrm{N})$ was carried out on a Perkin-Elmer Automatic Equipment Model 240.B.

\section{Synthesis of ligand:2,5-di(4-amino phenazonyl)} hexane

A solution of 4-amino phenazone (4.06 g. 20 mmole $)$ in absolute ethanol $(25 \mathrm{ml})$ was added to a solution of 2,5-hexandione $(1.14 \mathrm{~g}$, $10 \mathrm{mmol})$ in ethanol (15) ml.The reaction was Stirred and heated in water bath $(40-50){ }^{\circ} \mathrm{C}$ for six hr. after the addition of excess of Ethanol $(50 \mathrm{ml})$. A colorless precipitated and washed with diethyl ether .Recrystallization has been carried out in methanol. The product was dried via anhydrous $\mathrm{CaCl}_{2}$ in vacuum. The yield is (3.3g),83\%,mp. $198 \mathrm{C}^{\mathrm{o}}$.
Synthesis of $\mathrm{VO}(\mathrm{II}), \mathrm{Mn}(\mathrm{II}), \mathrm{Fe}(\mathrm{II}) \mathrm{Co}(\mathrm{II}), \mathrm{Ni}(\mathrm{II})$ $\mathrm{Cu}(\mathrm{II})$ and $\mathrm{Zn}$ (II) complexes:

A solution of metal salts $\left[\mathrm{VOSO}_{4}\right.$, $\mathrm{MnCl}_{2} \cdot 4 \mathrm{H}_{2} \mathrm{O}, \mathrm{FeCl}_{2}, \mathrm{Co}(\mathrm{OAc})_{2}$. $\quad 4 \mathrm{H}_{2} \mathrm{O}$, $\mathrm{Ni}(\mathrm{OAc})_{2} \quad 6 \mathrm{H}_{2} \mathrm{Cu}(\mathrm{OAc})_{2} \cdot \mathrm{H}_{2} \mathrm{O}$ and $\mathrm{ZnSO}_{4}$, ] in DMF (20mmol) was mixed with the Schiff base in DMF (20mmol, 5.628g) in a (1:1) molar ratio. The contents were refluxed in 100 $\mathrm{ml}$ of DMF in an oil bath for six to seven hr. The refluxed solution was poured into ice cold water. A colored solid product was separated and isolated by filtration. Then, was washed with ether. Recrystallization has been carried out in dimethyl sulfoxide and dimethyl formamide. The product was dried via anhydrous $\mathrm{CaCl}_{2}$ in vacuum at room temperature. The yield was in the range (75$83) \%$ for all the complexes with respect to the ligand. They decomposed at $288-300^{\circ} \mathrm{C}$.

\section{Results and Discussion}

The new Schiff base was synthesized by the condensation reaction of 4-amino phenazone and 2,5-hexandion as described in Scheme (1).

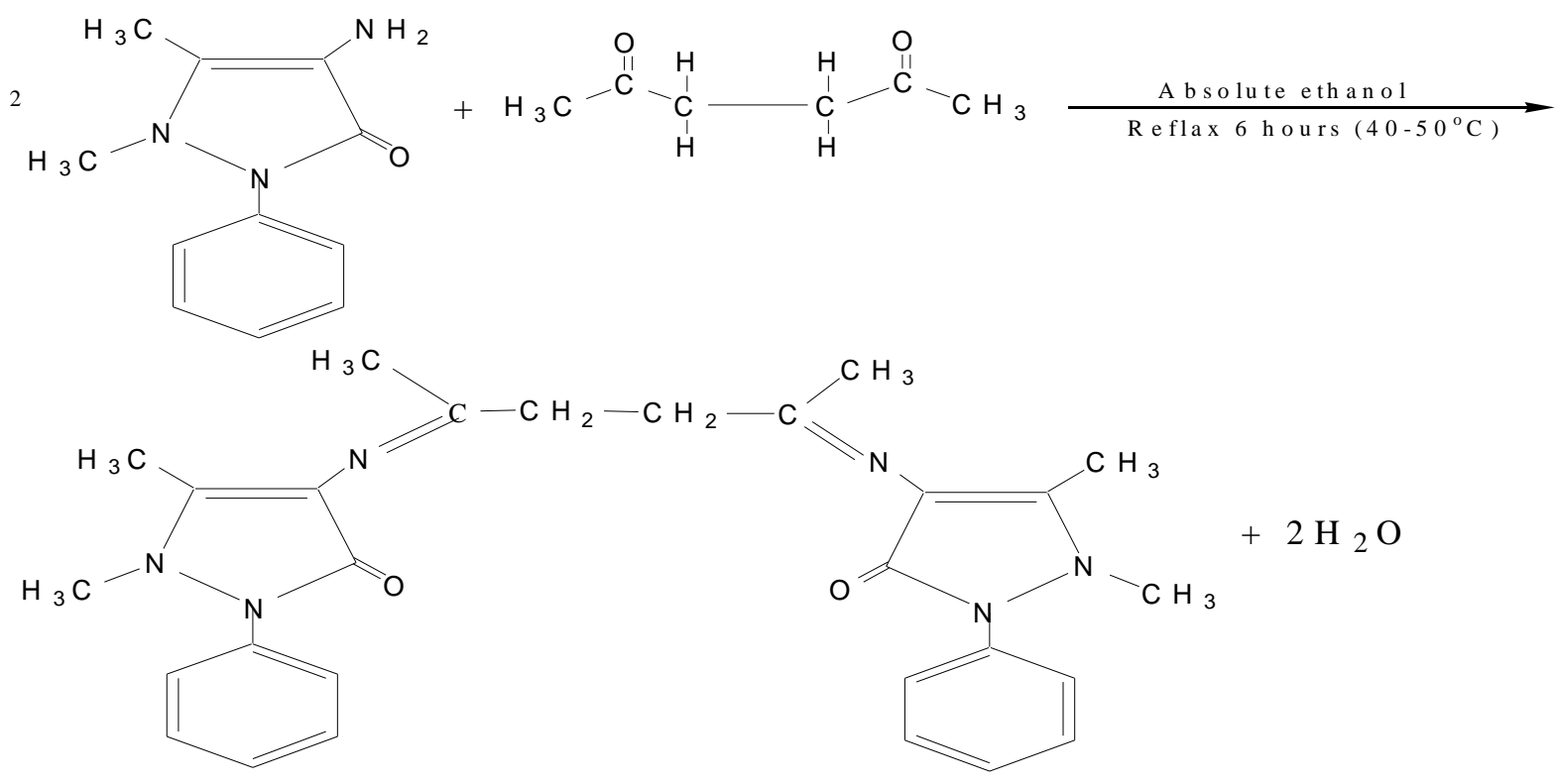

Scheme (1): Preparation of the Schiff Base (L).

Micro analytical, molar conductance and magnetic susceptibility data of the ligand and its complexes are given in Table (1). The stoichiometries of the ligand and its complexes were confirmed by their elemental analyses. The molar conductivity measurements for the complexes were recorded via using DMF in $10^{-3} \mathrm{M}$ solutions, which were in the range (169178) $\mathrm{ohm}^{-1} \cdot \mathrm{cm}^{2} \mathrm{~mol}^{-1}$. The results indicated to their electrolytic (1:2) behavior. ${ }^{(12,13)}$ The Metal-ligand Molar Ratio was found to be 
(1:1) according to Elemental Analysis (Fig.(1)).

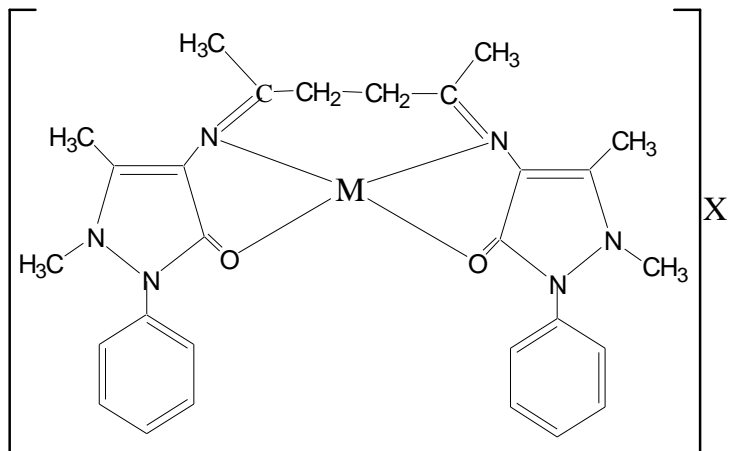

$\left(\mathrm{M}-\mathrm{X}=\mathrm{Co}(\mathrm{II})-(\mathrm{OAc})_{2}, \mathrm{Ni}(\mathrm{II})-(\mathrm{OAc})_{2}, \mathrm{Cu}(\mathrm{II})-(\mathrm{OAc})_{2}, \mathrm{VO}(\mathrm{II})\right.$ $\mathrm{SO}_{4}, \mathrm{Zn}(\mathrm{II})-\mathrm{SO}_{4}, \mathrm{Mn}(\mathrm{II})-\mathrm{Cl}_{2}$ or $\mathrm{Fe}(\mathrm{II})-\mathrm{Cl}_{2} ; \mathrm{OAc}=$ acetate $)$.

Fig.(1): Suggested structure as a tetrahedral for $\mathrm{Co}(\mathrm{II}), \mathrm{Ni}(\mathrm{II}), \mathrm{Mn}(\mathrm{II}), \mathrm{Fe}(\mathrm{II})$ and $\mathrm{Zn}(\mathrm{II})$ complexes square-planar for $\mathrm{Cu}(\mathrm{II})$ complex and square pyramidal for VO(II) complex.

The transition metal salts reactions with Schiff base are clarified in the following equations.

$\mathrm{L}+\mathrm{MX} . \mathrm{YH}_{2} \mathrm{O} \rightarrow[\mathrm{ML}] \mathrm{X} . \mathrm{H}_{2} \mathrm{O}$ where $\mathrm{M}, \mathrm{X}, \mathrm{Y}=\mathrm{VO}, \mathrm{SO}_{4,-} ; \mathrm{Mn}, \mathrm{Cl}_{2}, 4 ; \mathrm{Fe}, \mathrm{Cl}_{2},-;$ $\mathrm{Co},(\mathrm{OAc})_{2}, 4 ; \mathrm{Ni},(\mathrm{OAc})_{2}, 6 ; \mathrm{Cu},(\mathrm{OAc})_{2},-;$ and $\mathrm{Zn}, \mathrm{SO}_{4},-$.
${ }^{1} \mathrm{H}$ and. ${ }^{13} \mathrm{C}$-NMR spectra analysis:

A new synthesized ligand gave a satisfactory spectral data and the molecular structure was assigned on the basis of ${ }^{1} \mathrm{HNMR}$ and ${ }^{13}$ CNMR chemical shift. NMR spectra were determined in solution of $\left(\mathrm{CDCl}_{3}\right)$ with tetramethyl silane as an internal reference. The identification was using simple splitting patterns that were produced by the coupling of protons and carbons which they had a very different chemical shifts. according to the results obtained from the shift spectra, The molecular structure was illustrated as follows:

${ }^{13} \mathrm{C}-\mathrm{NMR}\left(\mathrm{CDCl}_{3}, \mathrm{ppm}, 400 \mathrm{MHz}\right)$ : $\delta 12.5(\mathrm{C}-1), \quad \delta 153.2(\mathrm{C}-2), \quad \delta 10.62(\mathrm{C}-3)$, $\delta 105.9(\mathrm{C}-4), \quad \delta 162.2(\mathrm{C}-5), \quad \delta 129.3(\mathrm{C}-6)$, $\delta 129.5(\mathrm{C}-7), \quad \delta 67.93(\mathrm{C}-8), \quad \delta 77.29(\mathrm{C}-9)$, $\delta 77.57(\mathrm{C}-10), \quad \delta 127.6(\mathrm{C}-11), \quad \delta 134.6(\mathrm{C}-$ 12), $\delta 124.3(\mathrm{C}-13), \delta 109.6(\mathrm{C}-14)$

${ }^{1} \mathrm{H}-\mathrm{NMR} \quad\left(\mathrm{CDCl}_{3}, \mathrm{ppm}, 400 \mathrm{MHz}\right): \delta 7.405-$ $7.52 \mathrm{ppm} \quad\left(2^{*} .2 \mathrm{H}, \mathrm{m}, \mathrm{a}-\mathrm{H}\right), \quad \delta 7.3-7.36 \mathrm{ppm}$ $\left(2^{*} .3 \mathrm{H}, \mathrm{m}, \mathrm{b}-\mathrm{H}\right), \delta 5.83-5.93 \mathrm{ppm}\left(2^{*} .2 \mathrm{H}, \mathrm{t}, 1-\mathrm{H}\right)$, $\delta 3.11-3.31 \mathrm{ppm}\left(2^{*} .3 \mathrm{H}, \mathrm{s}, 12-\mathrm{H}\right), \delta 2.04-2.14 \mathrm{ppm}$ $\left(2^{*} .6 \mathrm{H}, \mathrm{s}, 3-\mathrm{H}, 14-\mathrm{H}\right),{ }^{(14-16)}$

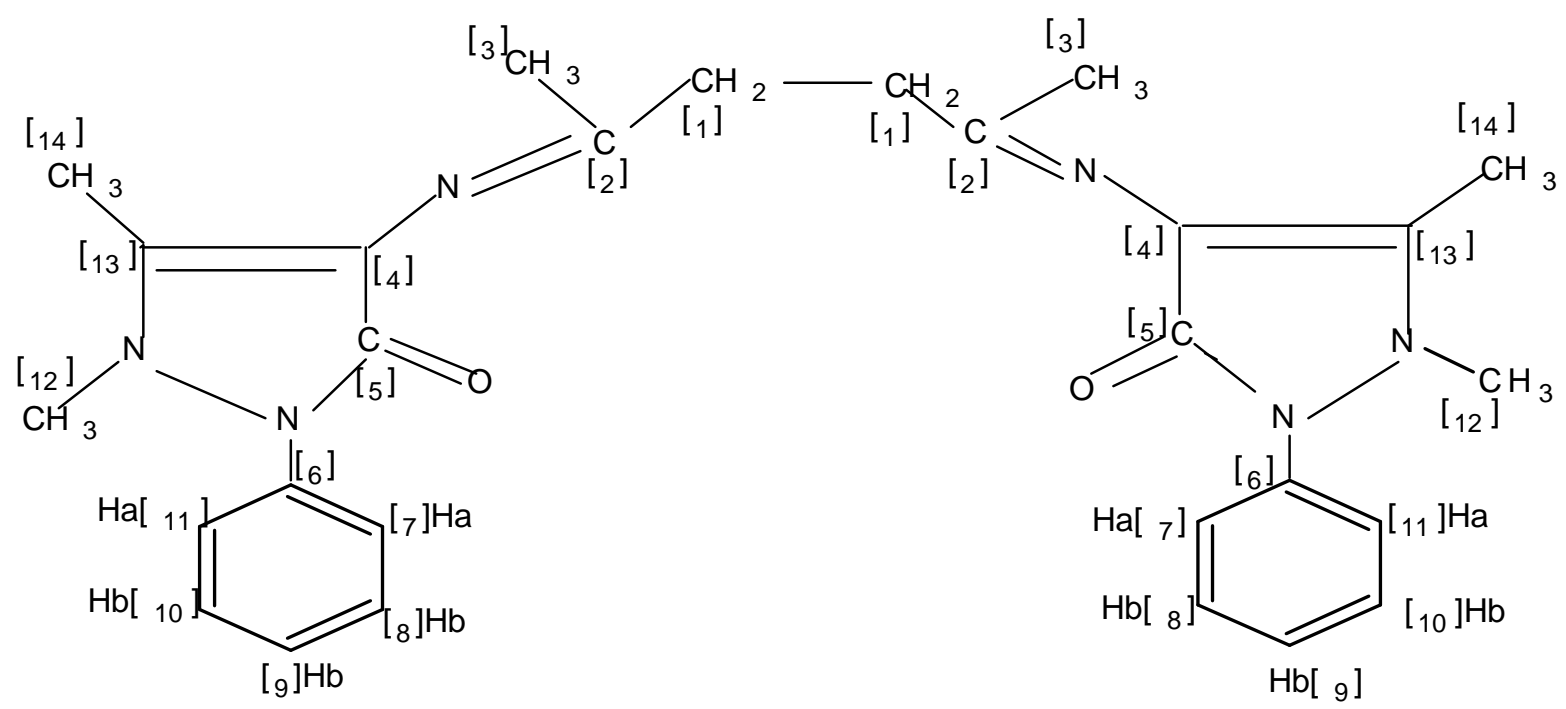




\section{Infrared Spectra:}

The characteristic stretching vibration modes concerning Schiff Base (L) and its metal complexes are described in Table (2).

The ligand (L) exhibited a strong high intensity bands appeared at (3035), (2920) and (1674) $\mathrm{cm}^{-1}$, which were ascribed to the stretching mode of $[\mathrm{vC}-\mathrm{H}$ aromatic, $v \mathrm{C}-\mathrm{H}$ aliphatic and $\mathrm{vC}=\mathrm{O}$ group respectively. Another strong bands appeared at 1620 and $1593 \mathrm{~cm}^{-1}$ were assigned to $\mathrm{vC}=\mathrm{N}$ group. ${ }^{(17)}$ as show in Table (2). Pertaining to the complexes, the Schiff base behaved as a tetradentate ligand, which were been coordinating with the metal via oxygen of carbonyl group and nitrogen of the azomethane group. The mentioned coordination was confirmed by shifting the bands $(\mathrm{vC}=\mathrm{O}$ and $\mathrm{vN}=\mathrm{C})$ to lower frequencies about (24-40) $\mathrm{cm}^{-1}$ and $(10-15,11-$ 14) $\mathrm{cm}^{-1}$ respectively. ${ }^{(18)}$ as clarified in Table (2).These observations were further indicated by the appearance of $(v M-N$ and $v(\mathrm{M}-\mathrm{O})$ respectively, Table (2). In the other hand, Acetate anion bands for [F,D and E] complexes were observed at $(1450,1446$, and 1440) $\mathrm{cm}^{-1}$ respectively which indicated to monodentate behavior of the mentioned anion. ${ }^{(19)}$ The $v(\mathrm{~V}=\mathrm{O})$ bands appeared very strong $965 \mathrm{~cm}^{-1}{ }^{(20)}$. Abroad band was observed around $(3450-3505) \mathrm{cm}^{-1}$ in each of $\mathrm{Mn}(\mathrm{II})$, $\mathrm{Co}(\mathrm{II}), \mathrm{Ni}(\mathrm{II})$ and $\mathrm{Cu}(\mathrm{II})$ complexes spectra, which is assigned to $\mathrm{vO}-\mathrm{H}$. The results led to a suggestion for the presence of water molecules in the complexes. ${ }^{(21)}$

\section{UV-Vis spectroscopic study:}

The Electronic Spectra of the metal complexes were recorded in their solutions in $10^{-4} \mathrm{M}$ DMF in the range $(200-1100) \mathrm{nm}$. The spectrum of free Schiff base ligand (L) showed strong peaks at (39062 and 33783) $\mathrm{cm}^{-1}$ attributed to $\left(\pi \rightarrow \pi^{*}\right.$ and $\left.\mathrm{n} \rightarrow \pi^{*}\right)$ respectively ${ }^{(22)}$.

For the cobalt complex, The measured Magnetic Moment was (4.56B.M) which is meant that the cobalt ion in its blue complex was paramagnetic with $d^{7}$ configuration in a distorted tetrahedral geometry ${ }^{(23)}$.The Electronic Spectrum for this complex showed broad peak at (14840-17505) $\mathrm{cm}^{-1}$, the peak was to the transitions ${ }^{4} \mathrm{~A}_{2} \rightarrow{ }^{4} \mathrm{~T}_{1}(\mathrm{P}) .{ }^{(24)}$

[E] $[\mathrm{Ni}(\mathrm{L})](\mathrm{OAc})_{2} \cdot \mathrm{H}_{2} \mathrm{O}$ compound, the presence of the absorption peaks at (17300 and $11049) \mathrm{cm}^{-1}$ which assign to the transition $\left.{ }^{3} \mathrm{~T}_{1} \rightarrow{ }^{3} \mathrm{~T}_{1} \mathrm{p}\right)$ and ${ }^{3} \mathrm{~T}_{1} \rightarrow{ }^{3} \mathrm{~A}_{2}$.

These transitions were characterized to the tetrahedral geometry around the $\mathrm{Ni}^{+2}$ ions. The value of the magnetic moment was 2.8 B.M, which confirmed the structure. $\mathrm{Dq}$ value was $450 \mathrm{~cm}^{-1(25,26)}$

[F] $[\mathrm{Cu}(\mathrm{L})](\mathrm{OAc})_{2} . \mathrm{H}_{2} \mathrm{O}$, The solution spectrum of the dark green complex exhibited intensive peaks at $(29850,27173$ and 20533) $\mathrm{cm}^{-1}$. The values of these peaks were in agreement with estimated values for highly distorted octahedral geometry. ${ }^{(27,28)}$ an effective magnetic moment at room temperature was (2.04B.M) which agreed with square planar geometry around $\mathrm{Cu}$ (II)complex. ${ }^{(29)}$. The green-yellow complex of $\mathrm{Mn}(\mathrm{II})$ showed strong bands at and the shoulder at $24213 \mathrm{~cm}^{-1}$ due to ${ }^{6} \mathrm{~A}_{1 \rightarrow}{ }^{4} \mathrm{~A}_{1+}{ }^{4} \mathrm{E}_{(\mathrm{G})}$ finally the band at $15337 \mathrm{~cm}^{-16} \mathrm{~A}_{1 \rightarrow}{ }^{4} \mathrm{~T}_{2}$, these values are accepted for tetrahedral complex ${ }^{(30)}$, the dark-green complex of iron(II) showed band at $31545 \mathrm{~cm}^{-1}$ belong to (LMCT) and another band at $22174 \mathrm{~cm}^{-1}$ caused by the electronic transition ${ }^{5} \mathrm{E} \rightarrow{ }^{5} \mathrm{~T}_{2}{ }^{(31)}$. The oxovanadium(IV) complex, electronic spectrum of shows three absorption bands at 26455,21645 and $13262 \mathrm{~cm}^{-1}$ respectively, which is consistent with a five-coordinate, square-pyramidal and is assigned to ${ }^{2} \mathrm{~B}_{2 \mathrm{~g}} \rightarrow 2 \mathrm{~A}_{1 \mathrm{~g}} \quad, \quad{ }^{2} \mathrm{~B}_{2 \mathrm{~g}} \rightarrow 2 \mathrm{~B}_{1 \mathrm{~g}}$ and $\quad{ }^{2} \mathrm{~B}_{2 \mathrm{~g}} \rightarrow 2 \mathrm{E}_{\mathrm{g}}$ transitions. The spectrum of $[\mathrm{ZnL}] \mathrm{SO}_{4}$, gave as absorption above $20833 \mathrm{~cm}^{-1}$, represents the internal ligand charge transfer. 
Table (1)

The Physical properties, and Elemental Analyses for the ligand and its metal complexes.

\begin{tabular}{|c|c|c|c|c|c|c|}
\hline \multirow[t]{2}{*}{ Compounds } & \multirow[t]{2}{*}{ F.Wt. g/mol } & \multirow[t]{2}{*}{$\boldsymbol{m} \cdot \boldsymbol{p}\left({ }^{o} \mathrm{C}\right)$} & \multirow[t]{2}{*}{ Yield (\%) } & $\begin{array}{l}\text { Elemental Analysis Calculated } \\
\text { (Found) \% }\end{array}$ & \multirow{2}{*}{$\begin{array}{l}\Omega 0 \\
{ }_{o h m^{-1}} \\
\mathrm{~cm}^{2} \cdot \mathrm{mol}^{1}\end{array}$} & \multirow{2}{*}{$\begin{array}{l}\boldsymbol{\mu} e f f \\
(\boldsymbol{B M})\end{array}$} \\
\hline & & & & C\% $\quad H \% \quad N \% \quad M \%$ & & \\
\hline $\begin{array}{l}\text { Ligand (L) } \\
\text { colorless } \\
\mathrm{C}_{28} \mathrm{H}_{32} \mathrm{~N}_{6} \mathrm{O}_{2} \\
\end{array}$ & 484 & 198 & 83 & 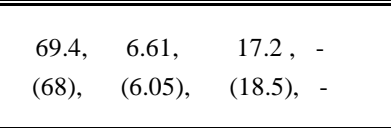 & - & - \\
\hline $\begin{array}{c}\mathrm{A}-[\mathrm{VOL}] \mathrm{SO}_{4} \\
\text { Pale-Green } \\
\mathrm{C}_{28} \mathrm{H}_{32} \mathrm{~N}_{6} \mathrm{O}_{7} \mathrm{SV}\end{array}$ & 665.348 & 305 & 78 & $\begin{array}{cccc}50.5, & 4.84, & 12.63, & 7.656 \\
(49.53) & (5.12) & 11.97) & (8.01)\end{array}$ & 173 & 1.71 \\
\hline $\begin{array}{c}\text { B-[MnL]Cl } l_{2} \\
\text { Green-yellow } \\
\mathrm{C}_{28} \mathrm{H}_{34} \mathrm{~N}_{6} \mathrm{O}_{3} \mathrm{Cl}_{2} \mathrm{Mn}\end{array}$ & 653.188 & 316 & 69 & $\begin{array}{l}51.487,4.899,12.866,8.41 \\
(52.09),(4.12),(11.68),(8.98)\end{array}$ & 169 & 4.65 \\
\hline $\begin{array}{c}\mathrm{C} \text {-[FeL }] \mathrm{Cl}_{2} \\
\text { Dark-green } \\
\mathrm{C}_{28} \mathrm{H}_{32} \mathrm{~N}_{6} \mathrm{O}_{2} \mathrm{Cl}_{2} \mathrm{Fe}\end{array}$ & 611.097 & 332 & 72 & $\begin{array}{c}55.03,5.236,13.75,9.138 \\
(55.99),(4.65),(12.76),(10.11)\end{array}$ & 177 & 4.7 \\
\hline $\begin{array}{c}\text { D- }[\mathrm{CoL}](\mathrm{OAc})_{2} . \mathrm{H}_{2} \mathrm{O} \\
\text { blue } \\
\mathrm{C}_{32} \mathrm{H}_{40} \mathrm{~N}_{6} \mathrm{O}_{7} \mathrm{Co}\end{array}$ & 705.93 & 292 & 75 & 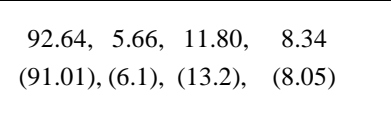 & 178 & 4.56 \\
\hline $\begin{array}{c}\text { E-[NiL] }(\mathrm{OAc})_{2} \cdot \mathrm{H}_{2} \mathrm{O} \\
\text { blue } \\
\mathrm{C}_{32} \mathrm{H}_{40} \mathrm{~N}_{6} \mathrm{O}_{7} \mathrm{Ni}\end{array}$ & 705.69 & 280 & 83 & $\begin{array}{c}92.67,5.66,11.03,8.31 \\
(93.15),(6.2),(11.8),(7.92)\end{array}$ & 172 & 2.8 \\
\hline $\begin{array}{c}\text { F- }[\mathrm{CuL}](\mathrm{OAc})_{2} . \mathrm{H}_{2} \mathrm{O} \\
\text { dark green } \\
\mathrm{C}_{32} \mathrm{H}_{40} \mathrm{~N}_{6} \mathrm{O}_{7} \mathrm{Cu}\end{array}$ & 710.5 & 300 & 78 & $\begin{array}{c}92.04,5.42,11.88,8.92 \\
(93.2),(4.98),(13.4),(9.98)\end{array}$ & 169 & 2.04 \\
\hline $\begin{array}{c}\text { F-[ZnL]SO } \mathrm{SO}_{4} \text { colorless } \\
\mathrm{C}_{28} \mathrm{H}_{32} \mathrm{~N}_{6} \mathrm{O}_{6} \mathrm{SZn}\end{array}$ & 645.798 & 288 & 88 & $\begin{array}{c}52.07,4.99,13.013,10.125 \\
(53.01),(5.07),(12.89),(10.01)\end{array}$ & 170 & Diamagnetic \\
\hline
\end{tabular}

Table (2)

The Characteristic infrared spectra bands for the ligand and its metal complexes.

\begin{tabular}{|c|c|c|c|c|c|c|c|}
\hline Compounds & $\begin{array}{l}\text { Water } \\
\text { vO-H }\end{array}$ & $\begin{array}{l}\text { vC-H arom } \\
\text { vC-H alph }\end{array}$ & $v C=O$ & $v C=N$ & $\begin{array}{c}v N-N \\
v A r-N\end{array}$ & $\begin{array}{l}v M-N \\
v M-O\end{array}$ & $\begin{array}{c}v M-O A c \\
v M-S_{4} \\
v M-C l\end{array}$ \\
\hline \multirow[t]{2}{*}{$(\mathbf{L})$} & - & 3035 & 1674 & 1620 & 1034 & - & - \\
\hline & & 2920 & & 1593 & 1321 & - & \\
\hline$[\mathrm{VOL}] \mathrm{SO}_{4}$ & - & $\begin{array}{l}3050 \\
2940\end{array}$ & 1650 & $\begin{array}{l}1600 \\
1580\end{array}$ & $\begin{array}{l}1030 \\
1322\end{array}$ & $\begin{array}{l}512 \\
486\end{array}$ & 1080 \\
\hline$[\mathrm{MnL}] \mathrm{Cl}_{2}$ & 3484 & $\begin{array}{l}3048 \\
2927\end{array}$ & 1648 & $\begin{array}{l}1602 \\
1579\end{array}$ & $\begin{array}{l}1039 \\
1324\end{array}$ & $\begin{array}{l}498 \\
450\end{array}$ & 405 \\
\hline$[\mathrm{FeL}] \mathrm{Cl}_{2}$ & - & $\begin{array}{l}3030 \\
2930\end{array}$ & 1646 & $\begin{array}{l}1604 \\
1581\end{array}$ & $\begin{array}{l}1033 \\
1321\end{array}$ & $\begin{array}{l}495 \\
442\end{array}$ & 392 \\
\hline$[\mathrm{CoL}](\mathrm{OAc})_{2} \cdot \mathrm{H}_{2} \mathrm{O}$ & 3450 & $\begin{array}{l}3040 \\
2920\end{array}$ & 1634 & $\begin{array}{l}1605 \\
1579\end{array}$ & $\begin{array}{l}1031 \\
1320\end{array}$ & $\begin{array}{l}510 \\
480\end{array}$ & 1446 \\
\hline$[\mathrm{NiL}](\mathrm{OAc})_{2} \cdot \mathrm{H}_{2} \mathrm{O}$ & 3470 & $\begin{array}{l}3045 \\
2915\end{array}$ & 1639 & $\begin{array}{l}1609 \\
1580\end{array}$ & $\begin{array}{l}1035 \\
1322\end{array}$ & $\begin{array}{l}519 \\
460\end{array}$ & 1440 \\
\hline$[\mathrm{CuL}](\mathrm{OAc})_{2} \cdot \mathrm{H}_{2} \mathrm{O}$ & 4505 & $\begin{array}{l}3038 \\
2924\end{array}$ & 1642 & $\begin{array}{l}1610 \\
1582\end{array}$ & $\begin{array}{l}1035 \\
1320\end{array}$ & $\begin{array}{l}495 \\
439\end{array}$ & 1450 \\
\hline$[\mathrm{ZnL}] \mathrm{SO}_{4}$ & - & $\begin{array}{l}3049 \\
2930\end{array}$ & 1645 & $\begin{array}{l}1609 \\
1583\end{array}$ & $\begin{array}{l}1032 \\
1324\end{array}$ & $\begin{array}{l}495 \\
442\end{array}$ & 1100 \\
\hline
\end{tabular}


Table (3)

Electronic Spectra in (DMF) Solvent for The Ligand and its Metal complexes.

\begin{tabular}{|c|c|c|c|c|c|}
\hline Compounds & $\lambda n m$ & $A B S$ & $v C m^{-1}$ & Transition & $\begin{array}{l}\text { Suggested } \\
\text { Structure }\end{array}$ \\
\hline$(\mathbf{L})$ & $\begin{array}{l}256 \\
296 \\
\end{array}$ & $\begin{array}{l}1.65 \\
2.24 \\
\end{array}$ & $\begin{array}{l}39062.5 \\
33783.7 \\
\end{array}$ & $\begin{array}{l}\pi \rightarrow \pi^{*} \\
\mathrm{n} \rightarrow \pi^{*}\end{array}$ & - \\
\hline$\left[\mathrm{VOL} \mathrm{SO}_{4}\right.$ & $\begin{array}{l}240 \\
310 \\
378 \\
462 \\
754 \\
\end{array}$ & $\begin{array}{l}1.12 \\
1.26 \\
2.13 \\
0.24 \\
0.41 \\
\end{array}$ & $\begin{array}{l}41666 \\
32258 \\
26455 \\
21645 \\
13262 \\
\end{array}$ & $\begin{array}{l}\text { Ligand field } \\
\text { Ligand field } \\
{ }^{2} \mathrm{~B}_{2 \mathrm{~g}} \rightarrow 2 \mathrm{~A}_{1 \mathrm{~g}} \\
{ }^{2} \mathrm{~B}_{2 \mathrm{~g}} \rightarrow 2 \mathrm{~B}_{1 \mathrm{~g}} \\
{ }^{2} \mathrm{~B}_{2 \mathrm{~g}} \rightarrow 2 \mathrm{E}_{\mathrm{g}}\end{array}$ & Square pyramidal \\
\hline$[\mathrm{MnL}] \mathrm{Cl}_{2}$ & $\begin{array}{l}274 \\
321 \\
413 \\
652 \\
\end{array}$ & $\begin{array}{l}1.36 \\
2.23 \\
0.07 \\
0.04 \\
\end{array}$ & $\begin{array}{l}36496 \\
31152 \\
24213 \\
15337 \\
\end{array}$ & $\begin{array}{l}\text { Ligand field } \\
\qquad \begin{array}{l}\mathrm{LMCT} \\
{ }^{6} \mathrm{~A}_{1 \rightarrow}{ }^{4} \mathrm{~A}_{1+}{ }^{4} \mathrm{E}_{(\mathrm{G})} \\
{ }^{6} \mathrm{~A}_{1 \rightarrow}{ }^{4} \mathrm{~T}_{2}\end{array}\end{array}$ & Tetrahedral \\
\hline$[\mathrm{FeL}] \mathrm{Cl}_{2}$ & $\begin{array}{l}275 \\
317 \\
451\end{array}$ & $\begin{array}{l}1.12 \\
1.97 \\
0.08 \\
\end{array}$ & $\begin{array}{l}36363 \\
31545 \\
22172 \\
\end{array}$ & $\begin{array}{l}\text { Ligand field } \\
\text { LMCT } \\
{ }^{5} \mathrm{E} \rightarrow{ }^{5} \mathrm{~T}_{2} \\
\end{array}$ & Tetrahedral \\
\hline$[\mathrm{CoL}](\mathrm{OAc})_{2} \cdot \mathrm{H}_{2} \mathrm{O}$ & $\begin{array}{l}263 \\
285 \\
340 \\
610\end{array}$ & $\begin{array}{l}1.05 \\
1.60 \\
2.12 \\
0.25\end{array}$ & $\begin{array}{c}38022.8 \\
35087.79 \\
29411.76 \\
16393\end{array}$ & $\begin{array}{l}\text { Ligand field } \\
\text { Ligand field } \\
\text { LMCT } \\
{ }^{4} \mathrm{~A}_{2} \rightarrow{ }^{4} \mathrm{~T}_{1}(\mathrm{P}) \\
\end{array}$ & Tetrahedral \\
\hline$[\mathrm{NiL}](\mathrm{OAc})_{2} \cdot \mathrm{H}_{2} \mathrm{O}$ & $\begin{array}{l}310 \\
407 \\
578 \\
905\end{array}$ & $\begin{array}{c}1.78 \\
1.9 \\
0.095 \\
0.05\end{array}$ & $\begin{array}{l}32258 \\
24570 \\
17300 \\
11049\end{array}$ & $\begin{array}{c}\text { Ligand field } \\
\text { LMCT } \\
{ }^{3} \mathrm{~T}_{1} \rightarrow{ }^{3} \mathrm{~T}_{1} \\
{ }^{3} \mathrm{~T}_{1} \rightarrow{ }^{3} \mathrm{~A}_{2}\end{array}$ & Tetrahedral \\
\hline$[\mathrm{CuL}](\mathrm{OAc})_{2} \cdot \mathrm{H}_{2} \mathrm{O}$ & $\begin{array}{l}262 \\
315 \\
335 \\
368 \\
487\end{array}$ & $\begin{array}{c}1.65 \\
2.34 \\
0.5 \\
0.19 \\
0.02\end{array}$ & $\begin{array}{l}38167 \\
31746 \\
29850 \\
27173 \\
20533 \\
\end{array}$ & $\begin{array}{l}\text { Ligand field } \\
\text { LMCT } \\
{ }^{2} \mathrm{~B}_{1 \mathrm{~g}} \rightarrow{ }^{2} \mathrm{~A}_{1 \mathrm{~g}} \\
{ }^{2} \mathrm{~B}_{1 \mathrm{~g}} \rightarrow{ }^{2} \mathrm{~B}_{2 \mathrm{~g}} \\
{ }^{2} \mathrm{~B}_{1 \mathrm{~g}} \rightarrow{ }^{2} \mathrm{E}_{\mathrm{g}}\end{array}$ & Square Planar \\
\hline$[\mathrm{ZnL}] \mathrm{SO}_{4}$ & $\begin{array}{l}260 \\
387 \\
480\end{array}$ & $\begin{array}{l}1.09 \\
1.64 \\
1.97\end{array}$ & $\begin{array}{l}38461.5 \\
25839.8 \\
20833.3\end{array}$ & $\begin{array}{l}\text { Ligand field } \\
\text { Ligand field } \\
\text { LMCT }\end{array}$ & Square Planar \\
\hline
\end{tabular}

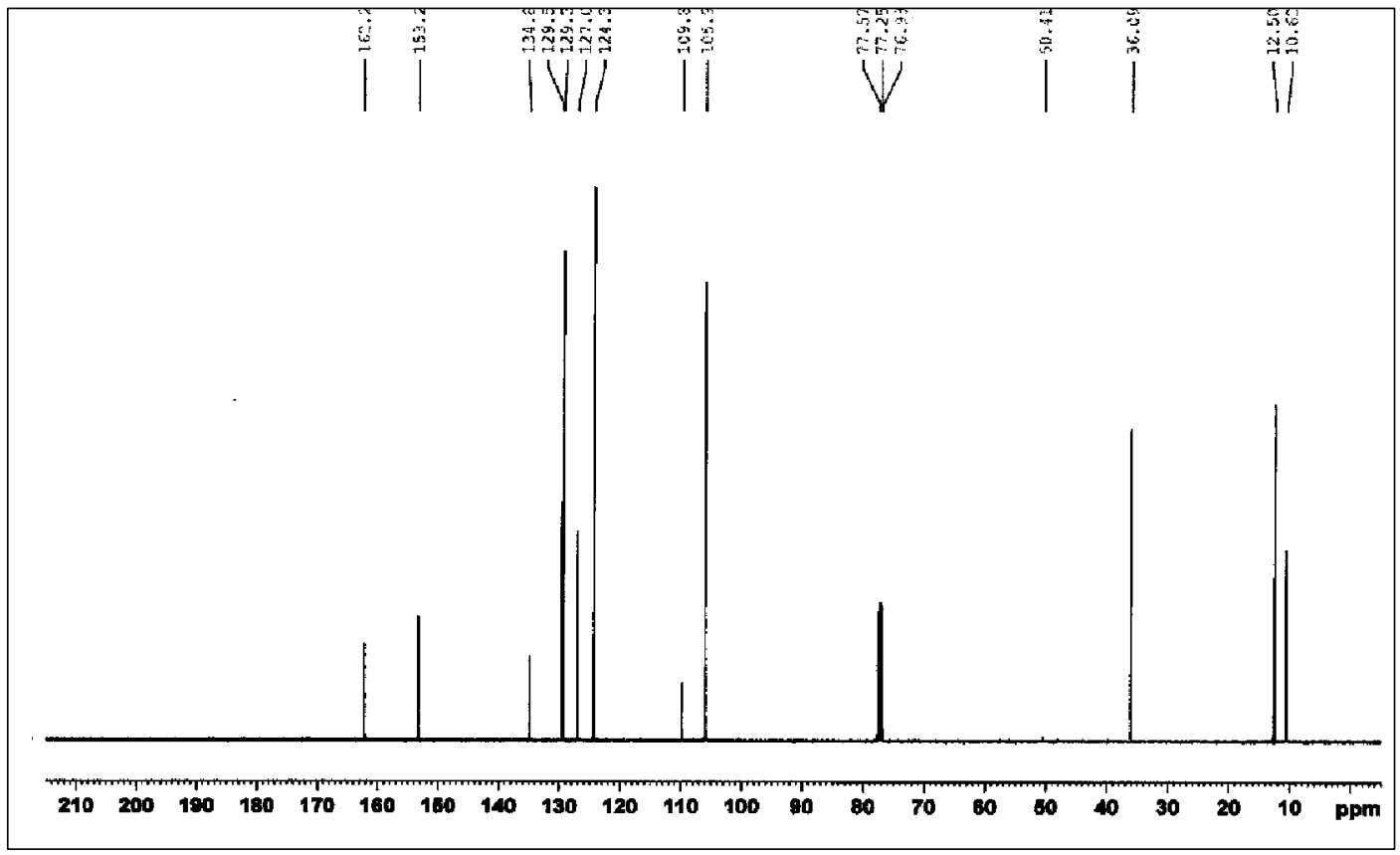

Fig. (1) : ${ }^{13} C$-NMR spectrum of the ligand $(L)$. 


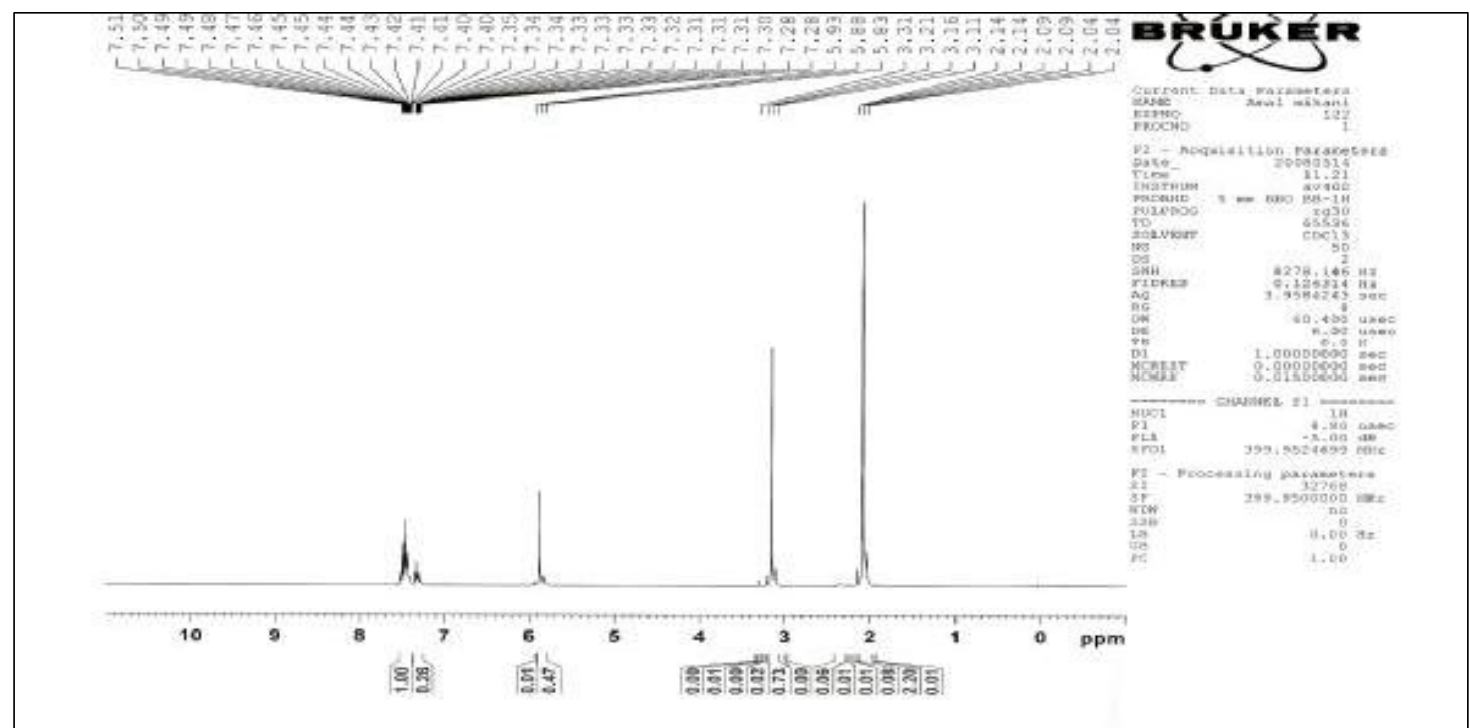

Fig. (2) : ${ }^{I} H$-NMR spectrum of the ligand $(L)$.

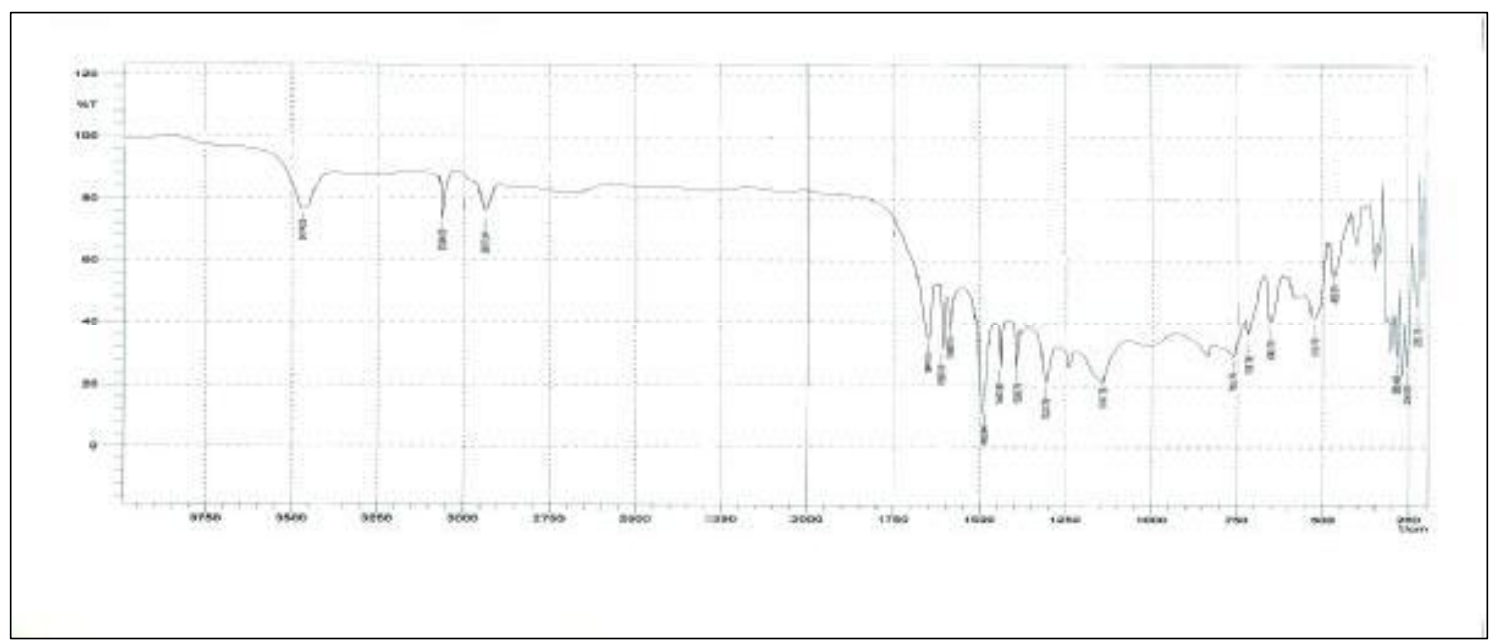

Fig. (3) : FT.IR spectrum for $[\mathrm{NiL}](\mathrm{OAc})_{2} . \mathrm{H}_{2} \mathrm{O}$ complex.

\section{References}

[1] H .Temel, S.Hben, M. Aslanoglu, A.Kihc and E.Tas, J. Chinese. Chem. Soc, 53, 2005.

[2] H.Temel, S.Hben, M.Aslanoglu, A.Kihc and E.Tas, J. Chinese. Chem. Soc, 53,5; 2006, 1027-1031.

[3] L.J.Klein, K. S.Alleman, D.G.Peters, J.A.Katy and J.P.Reilly, J. Electroanal. Chem, 481, 24, 2000.

[4] F. Azevedo, C.Freire and B.Castrode, Polyhedron, 211, 7, 1695, 2002.

[5] S.Zolezzi, E.Spodine and A.Decintim, Polyhedron, 21,1, 55, 2002.

[6] G.Turan-Zitouni, M.Sivaci, F. S.Kilic and K.Erol, Eur . J . Med . Chem ,36 , 685, 2001.
[7] S.Yoshioka, H.Ogata, T.Shibazaki and A.Ejima, Chem. Pharm .Bull , 29 , 1179, 1981.

[8] A. N.Evstopov, V. E.Yavorovskaya, E. S.Vorobev, Z. P.Kudonogova, L. N. Gritsenko, E. N.Schmidt, S. G.Medvedeva, D. V.Filimonov, T. P.Prishchep and A. S.Saratikov, J.Pharm.Chem, 26, 426, 1992.

[9] G. H.Sayed, A.Radwan, S. M.Mohamed, S.A.Shiba and M.Kalil, Ch .Chem ,10, 475, 1992.

[10] T.Cosmetic and A.Fragrance, J.Am. Coll. Toxicol, 11, 475, 1992.

[11] M.Verleye, I.Heurald and J. M. Gillardin, Pharmacol.Res, 41, 539, 2000.

[12] H.Temel, U.Cukir, V.Tolan, B.Otludil, H. L.Vgras, J. Coord .Chem, 57, 7, 571, 2004. 
[13] W. J.Geary, "The used conductivity measurements inorganic solvents for the characterization of coordination compounds", Coord.Chem.Rev, 1971, 7-81.

[14] V.Patel, M.Patel and R.Patel, J. Serb. Chem.Soc, 76, 2000,727-734.

[15] Y.Si, Q.Hu, Z.YHuang, G.ang and J.Yin, J.Turky.Chem,29,2005.

[16] R. M.Silverstein and F. X.Webster, "spectrometric identification of organic compounds", $6^{\text {th }} \mathrm{Ed}$, John Wiley and Sons, Inc, 1998, 217-232.

[17] M.Sonmez, J.Turki. Chem, 25, 2001.

[18] N.Nakamato, "Infrared and Raman Spectra of inorganic and Coordination Compounds", $4^{\text {th }}$ Ed Wiley, Inter Science, New York 1986.

[19] M.Parikh, "Absorption spectroscopy of Organic molecules", Addison. Wesley publishing Co. Inc, 1974.

[20] R. M.Silverstein, Clayton, G. B., and Morrill, T. C., "Spectrometric identification of organic compounds". $4^{\text {th }}$ Ed. John Wiley and sons, 1981.

[21] M.Sonmez and M.Sekerci, Polish. J. Chem, 76, 2002, 907-914.

[22] K.Burger, "Coordination Chemistry", Experimental Method, London BultWorths and Co(Publishers)Ltd, 1973.

[23] B. N.Figgis, "Introduction to ligand field". Inter science Publisher, Ins New York, 1966.

[24] T. M.Dunn, "The Visible and Ultraviolet Spectra of Complex Compounds in Modern Coordination Chemistry"., New York. Inter science, 1960.

[25] A. B.Lever, "Inorganic electronic spectroscopy", Elsevier Amsterdam,1984.

[26] G. J.Ballhausen, " Introduction to Ligand Field Theory" , Inter science, New York, 1966.

[27] N.Raman, J.D.Raja and A.Sakthivel, J.Chem.Sci, 119, 4, 2007, 303-310.

[28] S. N.Chio, R. D.Bereman, and J. R.Wassam, J. Inorganic. Nucl. Chem, 37, 2087, 1975.

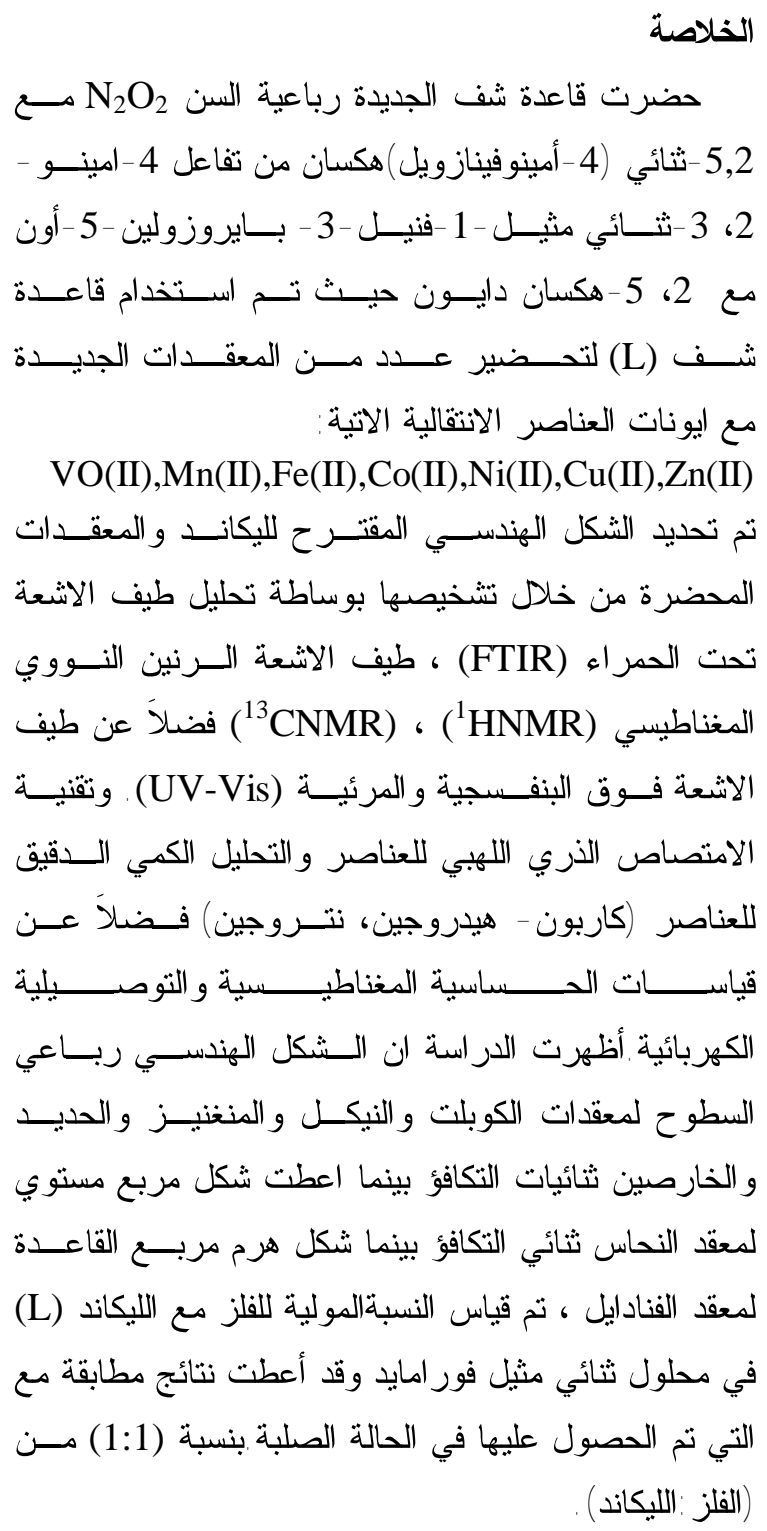

